\title{
Efectividad del programa "Anotaciones, que hablan por ti" en los conocimientos, actitudes y prácticas de los profesionales de enfermería en las anotaciones de enfermería según el modelo SOAPIE en los servicios de hospitalización del Hospital II Vitarte, Lima
}

\author{
Effectiveness of the program "Annotations, that speak for you" in the knowledge, attitudes and \\ practices of nursing professionals in nursing annotations according to the SOAPIE model in the \\ hospitalization services of Hospital II Vitarte, Lima
}

Edith Aguilar Campos ${ }^{\star *}$, Ángela Paredes Aguirre de Beltrán²

\begin{abstract}
RESUMEN
Objetivo: Determinar la efectividad del programa "Anotaciones, que hablan por ti" en los conocimientos, actitudes y prácticas de los profesionales de enfermería, en las anotaciones de enfermería según el Modelo SOAPIE, de los servicios de hospitalización del Hospital II Vitarte. Materiales y métodos: Estudio de enfoque cuantitativo; diseño pre experimental con un antes y después, de corte longitudinal. Participaron 24 profesionales de enfermería de los servicios de hospitalización de Medicina, Pediatría, Cirugía y Unidad de Vigilancia Intensiva; a quienes se les aplicó tres tipos de instrumentos: un cuestionario para medir conocimientos, la escala de actitudes tipo Likert y la guía de observación para evaluar la práctica. Resultados: El programa educativo mejoró el nivel de conocimientos sobre las anotaciones de enfermería en el modelo SOAPIE de los profesionales de enfermería, incrementando el promedio de calificación antes y después de la intervención de 20.7 a 25.0 puntos (p-valor $=.000$ ), asimismo, mejoró el nivel de actitudes, pasando de un promedio de 47.17 a 48.75 puntos ( $\mathrm{p}$-valor $=.000$ ) y mejoró la práctica de las anotaciones de enfermería, incrementando el promedio de puntuación de 6.33 a 10.21 (p-valor =.000). Conclusiones: El programa educativo "Anotaciones, que hablan por ti" fue efectivo en los conocimientos, actitudes y prácticas según el modelo SOAPIE, en los profesionales de Enfermería del Hospital II Vitarte-EsSalud.
\end{abstract}

Palabras clave: Conocimientos, actitudes y prácticas, Modelo SOAPIE, anotaciones de enfermería.

\begin{abstract}
Objective: To determine the effectiveness of the program "Annotations, which speak for you" in the knowledge, attitudes and practices of nursing professionals, in nursing notes according to the SOAPIE Model, of the hospitalization services of Hospital II Vitarte. Materials and methods: Quantitative study; Pre-experimental design with a before and after, longitudinal cut. Twenty-four nursing professionals from the hospitalization services of Medicine, Pediatrics, Surgery and Intensive Surveillance Unit participated; To whom three types of instruments were applied: a questionnaire to measure knowledge, the scale of attitudes Likert type and the guide of observation to evaluate the practice. Results: The educational program improved the level of knowledge about nursing annotations in the SOAPIE model of nursing professionals, increasing the average before and after the intervention from 20.7 to 25.0 points ( $\mathrm{p}$-value $=.000)$, $(\mathrm{P}$-value $=.000)$ and improved the nursing record practice, increasing the average score from 6.33 to 10.21 (p-value $=.6$ ). 000). Conclusions: The educational program "Anotaciones, que tallan por ti" was effective in the knowledge, attitudes and practices according to the SOAPIE model, in the Nursing professionals of Hospital II Vitarte-EsSalud.
\end{abstract}

Keywords: Knowledge, attitudes and practices, SOAPIE model, nursing annotations.

${ }^{1}$ Jefa del Departamento de enfermería, Hospital II Vitarte EsSalud, Lima, Perú.

${ }^{2}$ Directora de Unidad de Posgrado de Salud. Escuela de Posgrado. Universidad Peruana Unión, Lima Perú. 
Efectividad del programa "Anotaciones, que hablan por ti" en los conocimientos, actitudes y prácticas de los profesionales de enfermería en las anotaciones de enfermería según el modelo SOAPIE en los servicios de hospitalización del Hospital II Vitarte, Lima

\section{INTRODUCCIÓN}

El enfermero(a) es un profesional de la salud, con conocimiento científico y con arte manifiesto en los cuidados brindados a los pacientes de todas las edades, y estas son organizadas siguiendo diferentes pautas en su diseño y de acuerdo al tipo de paciente que se atiende.

El instrumento que utiliza el profesional de enfermería, en el cuidado de la persona se llama Proceso de Atención de Enfermería (PAE), el que comprende las anotaciones de enfermería. Estas anotaciones forman parte de la historia clínica y sirven de comunicación a nivel profesional, como interpersonal y, por lo tanto, se consigue el mayor nivel de calidad en la atención y posibilita el intercambio de información sobre los cuidados, mejorando la atención que presta el profesional de enfermería, permitiendo diferenciar su actuación con otros profesionales.

En este mundo globalizado, con la nueva tecnología sofisticada en todos los aspectos, ha traído muchos enfoques como la calidad total, la competitividad, la productividad, obligando al sector salud entre ellos a los profesionales de enfermería, a tener respuestas de cambio inmediatas con la finalidad de ofrecer al usuario un servicio de calidad comprobada resultante de un proceso de coordinación entre los actores. Por lo tanto, la documentación que maneja debe contar con los indicadores de calidad reconocidos internacionalmente.

La preocupación, en otros países, sobre la calidad de los registros de enfermería, es evidente, resaltado en el trabajo de investigación por Hernández-Corral, y Hernández -Mayen (2010), en las de Blascetta y Guzmán (2007), quienes ponen de manifiesto que las anotaciones de enfermería se convirtieron, en una rutina, una formalidad realizada por obligación y no como algo inherente a la función.

En el Perú, en la Ley del Trabajo de la Enfermera (o) No 27669, en el artículo 4, menciona que la enfermera se encarga del cuidado integral de las personas en todas las etapas de la vida y que será registrado obligatoriamente en la historia clínica del paciente $y / o$ en la ficha familiar.

El Ministerio de Salud- Oficina General de Epidemiología OGE (2001) ha elaborado indicadores Hospitalarios y de Gestión, en el Capítulo XIX, los que corresponden a Indicadores de enfermería y los específicos para nuestro trabajo son: Indicador 212: Porcentaje de Diagnósticos de Enfermería Registrados e Indicador 213: Calidad del Registro de
Enfermería, esta medición se basa en la existencia de un plan de atención estructurado según las normas establecidas en el SOAPIE.

Sin embargo, estas no se están cumpliendo, tal como resalta el trabajo de investigación, realizado por Fretel (2004), quien mencionó que el contenido de las anotaciones de enfermería revisadas solo se basan en términos generales sobre el estado del paciente, siendo los cuidados brindados en su mayoría al área física, encontrándose datos escasos en el área social, psicológica y espiritual. En el Hospital II Vitarte, Aguilar (2008), concluyó que de las 347 historias clínicas analizadas, solo el $20.7 \%$ registra en forma completa los datos subjetivos, el $15.9 \%$ registra en forma completa el análisis y valoración diagnóstica. El $80.1 \%$ no registra la planificación, el $82.1 \%$ registra en forma parcial la variable intervención y el $94.5 \%$ registra en forma parcial la evaluación de las actividades, del mismo modo; Castillo (2011), concluyó que el conocimiento y práctica en el Modelo SOAPIE, se relaciona en mínimo porcentaje con la estructura de las anotaciones de enfermería, así mismo el nivel de conocimiento se relaciona débilmente con el contenido de estas a diferencia de las practica donde la relación es muy fuerte en el contenido de las anotaciones.

En las auditorias de enfermería, realizadas en los servicios de hospitalización del Hospital II Vitarte, se evidencia persistencia de anotaciones de enfermería sin aplicación del modelo SOAPIE.

Observada esta situación, se hace necesario presentar un programa educativo y determinar la efectividad del programa educativo "Anotaciones, que hablan por ti” en los conocimientos, actitudes y prácticas de los profesionales de enfermería, en las anotaciones de enfermería, según el Modelo SOAPIE, de los servicios de hospitalización del Hospital II Vitarte.

Se consideró como hipótesis alterna : El programa educativo "Anotaciones, que hablan por ti" mejora los conocimientos, actitudes y prácticas de los profesionales de enfermería en las anotaciones de enfermería, según el modelo SOAPIE de los servicios de hospitalización del Hospital II Vitarte y como hipótesis nula: El programa Educativo "Anotaciones, que hablan por ti" no mejora los conocimientos, actitudes y prácticas de los profesionales de enfermería en las anotaciones de enfermería, según el Modelo SOAPIE de los servicios de hospitalización del Hospital II Vitarte.

Se consideró como variable independiente, al programa educativo "Anotaciones, que hablan por ti" 
y como variables dependientes a los conocimientos, actitudes y prácticas.

\section{MATERIAL Y MÉTODOS}

Es un estudio con enfoque cuantitativo, porque hace uso de los modelos estadísticos para la presentación de los resultados. Es de diseño pre experimental con pre y post test, con una sola muestra, no aleatorizado. Se dice que es pre experimental porque se analiza el efecto que produce la variable independiente sobre la variable dependiente y el control es el mismo sujeto que participa en el experimento. El modelo del diseño es el siguiente:

$\mathbf{G} \quad \mathbf{O}_{0} \quad \mathbf{X} \quad \mathbf{O}_{1}$
Dónde:
G: Grupo de estudio
$\mathbf{O}_{\mathbf{0}}$ : Pre test
$\mathbf{X}$ : Variable tratamiento (Programa)
$\mathbf{O}_{\mathbf{1}}$ : Pos test

\section{Participantes}

Se utilizó el muestreo no probabilístico, por conveniencia, por la disponibilidad para colaborar con el estudio, participaron 25 profesionales de enfermería de los servicios de hospitalización de Medicina (08), Pediatría (05), Cirugía (07) y Unidad de Vigilancia Intensiva (05).

Durante la transcripción de datos se detectó que un participante, no contestó el instrumento de Likert, por lo tanto, se decidió continuar el estudio con 24 participantes.

De los 24 profesionales de enfermería que participaron en el estudio, el $85.5 \%$ tienen una edad de 41 años a más y el $12.5 \%$ entre las edades de 31 y 40 años; el 29.2\% labora en el Servicio de Medicina y Cirugía, $20.8 \%$ al Servicio de Pediatría y UVI; respecto a la experiencia profesional el 50\% afirmó tener entre 11 y 20 años de experiencia, el $41.7 \%$ más de 20 años y el $8.3 \%$ entre 6 y 10 años.

\section{Instrumentos}

Para la recolección de los datos se elaboró tres instrumentos.

\section{Cuestionario de conocimientos:}

La investigadora elaboró el cuestionario en base a trabajos de investigación y bibliografía de registros de enfermería, el cual permitió identificar el nivel de conocimientos del Modelo SOAPIE aplicado en las anotaciones de enfermería.

El cuestionario comprende de 17 preguntas las cuales fueron valoradas con 2 puntos para los ítems que fueron respondidos de manera correcta y 0 puntos para los ítems que fueron respondidos de manera errada. Para la valoración final de la variable se tomó en cuenta los siguientes niveles: bajo (0-18), regular (20-26), y alto (28-34).

\section{Escala de actitudes tipo Likert:}

Para la construcción de los ítems de la variable actitud, se basó en diferentes literaturas relacionadas al SOAPIE. La medición de esta variable se realizó a través de 11 ítems medidos con la escala Likert siguiente: totalmente de acuerdo, de acuerdo, indiferente, en desacuerdo y totalmente en desacuerdo dichas categorías fueron valoradas con las puntuaciones $5,4,3,2,1$ respectivamente. La valoración final de la variable cuenta con las siguientes categorías: Actitud totalmente desfavorable (11-22), actitud medianamente desfavorable (23-33), actitud medianamente favorable (34-44) y actitud totalmente favorable (45-55); las dos primeras categorías se conocen como actitud negativa y las dos últimas como actitud positiva.

\section{Guía de observación:}

Comprende afirmaciones orientadas a medir la aplicabilidad del Modelo SOAPIE, la guía de observación, está dividido en 11 ítems y seis dimensiones que validan la aplicación del PAE, cada ítem es valorado de acuerdo a las categorías: si aplica, aplica parcialmente y no aplica; las puntuaciones de cada categoría fueron 2, 1 y 0 respectivamente. La valoración final de las prácticas fue la siguiente: prácticas adecuadas (17.51 - 22), prácticas parcialmente adecuadas $(10.51-17.50)$, y prácticas inadecuadas $(0-10.50)$.

La validación de los instrumentos fue por Docentes de la Universidad Peruana Unión y profesional de enfermería asistenciales del Hospital II Vitarte EsSalud. El instrumento que recopilaba información general y sobre los conocimientos y actitudes, fue aplicado mediante la técnica de la encuesta. La técnica para la recolección de los datos sobre las prácticas basadas en el modelo SOAPIE en las anotaciones de 
Efectividad del programa "Anotaciones, que hablan por ti" en los conocimientos, actitudes y prácticas de los profesionales de enfermería en las anotaciones de enfermería según el modelo SOAPIE en los servicios de hospitalización del Hospital II Vitarte, Lima

enfermería fue la revisión de las notas de enfermería, evaluando a cada una de ellas si cumplían o no con las especificaciones del instrumento.

Los datos recolectados fueron procesados con el programa estadístico SPSS, versión 23, se tomaron en cuenta los valores de cada categoría para el ingreso de los datos al software; se utilizaron las opciones de suma y recodificación del paquete estadístico en la fase de preparación.
Después de haber sido preparado los datos se presentaron los resultados a través de tablas de resumen con las medidas más representativas de cada resultado. Para realizar la prueba de hipótesis se consideró un nivel de significancia del 5\%, es decir, un nivel de confianza del $95 \%$. Se consideró por conveniente realizar la comprobación de la hipótesis a través de la prueba no paramétrica de rangos de Wilcoxon, por tratarse de una muestra pequeña, además se realizó la aproximación a $\mathrm{Z}$.

\section{RESULTADOS}

Tabla 1

Nivel de conocimiento de los profesionales de enfermería del Hospital II Vitarte EsSalud respecto a las anotaciones de enfermería bajo el modelo SOAPIE, Lima.

\begin{tabular}{ccccc}
\hline Conocimiento & \multicolumn{2}{c}{ Pre test } & \multicolumn{3}{c}{ Post test } \\
\cline { 2 - 5 } & $\mathrm{n}$ & $\%$ & $\mathrm{n}$ & $\%$ \\
\hline Bajo & 7 & 29.2 & 3 & 12.5 \\
Regular & 17 & 70.8 & 10 & 41.7 \\
Alto & 0 & 0.0 & 11 & 45.8 \\
\hline
\end{tabular}

La tabla 1 muestra que el $70.8 \%$ presentó un nivel de conocimiento regular, y el $29.2 \%$ nivel de conocimiento bajo en la primera medición; después de la aplicación del programa el $45.8 \%$ presento nivel de conocimiento alto, 41.7 conocimiento regular y el $12.5 \%$ conocimiento bajo.

Tabla 2

Actitud de los profesionales de enfermería del Hospital II Vitarte EsSalud respecto a las anotaciones de enfermería bajo el modelo SOAPIE, Lima.

\begin{tabular}{lllll}
\hline Actitud & Pre test & \multicolumn{3}{c}{ Post test } \\
\cline { 2 - 5 } & $\mathrm{n}$ & $\%$ & $\mathrm{n}$ & $\%$ \\
\hline Actitud Negativa & 1 & 4.2 & 0 & 0.0 \\
Actitud Positiva & 23 & 95.5 & 24 & 100.0 \\
\hline
\end{tabular}

La Tabla 2 muestra que antes de la aplicación del programa un $95.2 \%$ de profesionales presentó una actitud positiva y el $4.2 \%$ actitud negativa; después del pos test el $100 \%$ presentó actitud positiva.

Tabla 3

Práctica de los profesionales de enfermería del Hospital II Vitarte EsSalud respecto a las anotaciones en enfermería bajo el modelo SOAPIE, Lima.

\begin{tabular}{ccccc}
\hline Práctica & \multicolumn{2}{c}{ Pre test } & \multicolumn{2}{c}{ Post test } \\
\cline { 2 - 5 } & $\mathrm{n}$ & $\%$ & $\mathrm{n}$ & $\%$ \\
\hline Inadecuadas & 21 & 87.5 & 14 & 58.3 \\
Parcialmente adecuadas & 3 & 12.5 & 10 & 41.7 \\
Adecuadas & 0 & 0.0 & 0 & 0.0 \\
\hline
\end{tabular}

La tabla 3 muestra que el $87.5 \%$ realizaba prácticas inadecuadas y un $12.5 \%$ prácticas parcialmente adecuadas antes de la implementación del programa; sin embargo, después de su implementación el porcentaje de profesionales con prácticas inadecuadas se redujo a $58.3 \%$ y el $41.7 \%$ presentó prácticas parcialmente adecuadas. 
Tabla 4

Prueba de hipótesis de la efectividad del programa "Anotaciones que hablan por ti" en los conocimientos, actitudes y prácticas del profesional de enfermería del Hospital II Vitarte EsSalud, Lima.

\begin{tabular}{lllllll}
\hline Variables y momentos & $(\mathrm{S})$ & $\mathrm{Me}(\mathrm{RI})$ & $\mathrm{R}-$ & $\mathrm{R}+$ & $\mathrm{Z}$ & $\mathrm{p}$ \\
\hline Conocimientos & & & & & & \\
$\quad$ Pre test & $20.75(4.78)$ & $22(6)$ & 0 & 136 & -3.534 & 0.000 \\
$\quad$ Post test & $24.92(4.45)$ & $26(7.5)$ & & & & \\
$\quad$ Actitud & & & & & \\
$\quad$ Pre test & $47.17(5.97)$ & $44(7.75)$ & 0 & 120 & -3.433 & 0.000 \\
$\quad$ Post Test & $48.75(4.55)$ & $48.5(6.75)$ & & & & \\
Práctica & & & & & & \\
$\quad$ Pre test & $6.33(2.36)$ & $6(3.05)$ & 5 & 225 & -4.143 & 0.000 \\
$\quad$ Post test & $10.21(3.24)$ & $10.20(4.85)$ & & & & \\
\hline
\end{tabular}

La Tabla 5 presenta las medidas de resumen más representativas y la comparación de las puntuaciones obtenidas en el pre test y post test para las variables en estudio; se muestran los resultados de la prueba de rangos de Wilcoxon y la aproximación a la distribución $\mathrm{Z}$ por tratarse de muestras superiores a 20 (Pértega y Pita, 2007), evidenciándose diferencias estadísticamente significativas entre las puntuaciones del pre test y post test para las variables conocimiento $(\mathrm{Z}=-3.534, \mathrm{p}=0.000)$, actitud $(\mathrm{Z}=-3.433, \mathrm{p}=0.000) \mathrm{y}$ prácticas $(\mathrm{Z}=-4.143, \mathrm{p}=0.000)$.

\section{DISCUSIÓN}

El estudio para determinar la efectividad del programa educativo "Anotaciones, que hablan por ti” en los conocimientos, actitudes y prácticas de los profesionales de enfermería, en las anotaciones de enfermería según el Modelo SOAPIE, de los servicios de hospitalización del Hospital II Vitarte, demostró que el programa educativo aplicado, mejoró el nivel de conocimientos de las profesionales de enfermería, incrementó de una puntuación promedio de 20.75 en el test de conocimientos inicial a una puntuación promedio de 24.92 en el test final, considerándose estás mejoras como estadísticamente significativas. Estos resultados concuerdan con los encontrados por Pecho, M. (2003), en su estudio sobre "Efectividad de un programa educativo en el mejoramiento de conocimiento y calidad de las anotaciones de enfermería en el Hospital Regional de Ica”, en la que para una muestra de 60 enfermeras, encontró que los resultados fueron altamente significativos después de aplicado el programa, evidenciando un mejor conocimiento, comprobando así la efectividad del programa.

Considerando que vivimos en una era de constantes cambios y descubrimientos científicos en todas las áreas del saber y en especial de salud, es importante que los profesionales de enfermería posean conocimientos para cuidar mejor al paciente y tomar decisiones adecuadas con un buen sustento científico. Polit y Hungler (1987) plantearon que la meta final de cualquier profesión es mejorar la práctica de sus miembros, en ese sentido, Enfermería busca mejorar su imagen y como tal se dedica a la adquisición constante de un conjunto de conocimientos científicos que permitan realizar su práctica con eficacia, y precisen el efecto que tienen sus conocimientos sobre la persona y sociedad. Por tal razón, el programa educativo "Anotaciones que hablan por ti" busca dar a conocer y reforzar los conocimientos del Proceso de Atención de enfermería con el modelo SOAPIE.

Así mismo, hubo mejoras estadísticamente significativas en cuanto al cambio en el nivel de actitudes de los profesionales de enfermería antes y después de la intervención del programa educativo, pasando de una puntuación promedio de 47.17 en el test inicial a una puntuación de 48.75 en el test final, considerado como positiva. Estos resultados concuerdan con los hallados por Reyes, Jara y Merino (2007), en su trabajo "Adherencia de las enfermeras a utilizar un modelo teórico como base de la valoración de enfermería", encontrando que la adherencia, medida por la actitud, fue aceptada con una asociación estadísticamente significativa.

Se debe mencionar que no concuerda con nuestra investigación los resultados obtenidos en el trabajo de tesis de Quino (2014), quien encontró que el $65 \%$ poseen actitud indecisa frente a la utilidad y aplicabilidad de notas de enfermería modelo SOAPIE.

Con respecto a la práctica de los profesionales de enfermería, antes y después de la intervención, 
Efectividad del programa "Anotaciones, que hablan por ti" en los conocimientos, actitudes y prácticas de los profesionales de enfermería en las anotaciones de enfermería según el modelo SOAPIE en los servicios de hospitalización del Hospital II Vitarte, Lima

la muestra tuvo un incremento de $61.99 \%$ en su puntuación promedio, de 6.33 a 10.21 , considerando un incremento estadísticamente significativo. Estos resultados se asemejan con los hallazgos de Reyes, Jara y Merino (2007), en su trabajo "Adherencia de las enfermeras a utilizar un modelo teórico como base de la valoración de enfermería", en la que concluye que si bien se obtuvieron resultados positivos en términos cognitivos, los cambios en cuanto a la práctica no mostró diferencias significativas. Asimismo, el estudio de Hernández-Corral (2010), "Repercusión de un programa de mejora continua en los registros de enfermería", desarrollado en México, demostró cambios en la eficiencia global (IEG) de registro de enfermería de 5.91, en la puntuación global, a 6.96 después del programa de mejora continua, encontrando estos cambios como estadísticamente significativos.

Así mismo, García y Sánchez (2013), en el trabajo de investigación cuantitativa "Diseño e implementación de Taller Teórico-Práctico sobre Proceso de enfermería y registro diario S.O.A.P.I.E corroboran que, hubo un aumento significativo de la puntuación, ya que antes del taller la puntuación era de 3.3 puntos $(13.2 \%)$, pero después de la instrucción recibida aumenta a 14 puntos (56\%), siendo el incremento significativo de 10.7 puntos $(42.8 \%)$ resultando la diferencia significativa $(\mathrm{p}<0.001)$ según el valor conseguido del estadístico de decision de T de Student.

Rojas (2010) ratificó con los resultados de la investigación titulada "Eficacia de un programa educativo, en la calidad técnica de las anotaciones de enfermería en un servicio de medicina interna de un hospital nacional. Lima - Perú. 2014", en la que concluyó que, el programa educativo mejora la calidad en la dimensión contenido de las anotaciones de enfermera con el modelo SOAPIE y fue eficaz en el incremento de la calidad técnica de las anotaciones de enfermería del servicio de medicina interna $6 \mathrm{C}$ del Hospital Nacional Edgardo Rebagliati Martins, pasando de un nivel de calidad deficiente a un nivel de "buena" calidad. De igual modo, el estudio de Pecho (2003), evidenció la efectividad del programa en el nivel de calidad de las anotaciones de enfermería, después de la intervención de un programa educativo.

Diferentes estudios transversales concuerdan con los hallazgos de este estudio acerca de la deficiente calidad de las anotaciones de enfermería antes de la intervención de un Programa Educativo, así, el estudio de Palomino, B. (2010) "Calidad de las anotaciones de enfermería en el Hospital Regional de Ayacucho", observó que el $73.08 \%$ de las anotaciones de enfermería tenían deficiente calidad, referidas a la dimensión contenido. El estudio de Fretel, (2004),
"Evaluación de las Anotaciones de Enfermería en el 2000-2002 en el Servicio de Neurología del Hospital Guillermo Almenara" encontró que solo el 21.8 $\%$ de las anotaciones de enfermería fue adecuada, indicando que no se considera, en sus criterios de elaboración, datos importantes en base al PAE.

Se considera también que en el trabajo de tesis de Quino(2014),'Conocimiento y Actitud sobre notas de enfermería Modelo SOAPIE en enfermeras del Hospital Regional Manuel Núñez Butrón, Puno concluyó concluye que los resultados obtenidos fueron que el $50 \%$ de las enfermeras(os) poseen conocimiento regular sobre notas de enfermería Modelo SOAPIE y solo el 38\% tiene el conocimiento bueno, referente al conocimiento sobre aspectos de las notas de enfermería modelo SOAPIE, predomina el conocimiento de bueno en presentación e identificación de quien realiza las notas y el conocimiento deficiente y regular en el diagnóstico de enfermería. La relación establecida entre las variables, se demuestra porcentual y estadísticamente con la prueba Chi Cuadrado donde X2 c=10.00> $\mathrm{X} 2 \mathrm{t}=9.49$, por lo tanto, existe relación significativa entre el nivel de conocimiento y actitud sobre Notas de Enfermería modelo SOAPIE de Enfermeras del Hospital Regional Manuel Núñez Butrón -Puno.

Cabe resaltar que en el Código de ética y Deontología del Colegio de Enfermeras(os) del Perú DL 22315 Capítulo III Artículo 27, menciona lo siguiente: "La Enfermera(O) debe ser objetiva y veraz en sus informes, declaraciones, testimonios verbales $y$ escritos, relacionados con su desempeño profesional", sustenta la necesidad de registrar los cuidados de forma científica.

La práctica adecuada del uso de anotaciones de enfermería con el modelo SOAPIE, va a permitir mejorar la calidad de atención del usuario, porque se cuenta con una fuente de información clara, legible, confiable, exacta que permite tomar decisiones para el bienestar y mejora de la salud del paciente, y se constituye en un documento legal y de investigación.

Por lo tanto, las conclusiones del programa educativo "Anotaciones, que hablan por ti", mejoró el nivel de conocimientos sobre las anotaciones de enfermería en el modelo SOAPIE de los profesionales de enfermería de los servicios de hospitalización del Hospital II Vitarte, incrementando el promedio de notas antes y después de la intervención de 20.75 a 24.92 puntos.

Asimismo, mejoró nivel de actitudes en el modelo SOAPIE, de los profesionales de enfermería de los servicios de hospitalización del Hospital II Vitarte, 
antes y después de la intervención, pasando de un promedio pre test de 47.17 y después del test a 48.75 considerado como nivel positivo.

De igual manera, mejoró el nivel de práctica de los profesionales de enfermería de los servicios de hospitalización del Hospital II Vitarte, antes y después de la intervención, pasando de un promedio de 6.33 a 10.21 .

Como conclusión final, se puede afirmar que el programa educativo "Anotaciones, que hablan por ti" fue efectivo en la mejora del nivel de conocimientos, actitudes y prácticas en las anotaciones de enfermería según el modelo SOAPIE en los diferentes servicios de hospitalización, concluyendo que se acepta la hipótesis alterna.

\section{Declaración de financiamiento y de conflicto de intereses:}

El estudio fue financiado por los autores, quienes declaran no tener algún tipo de conflicto de interés en la investigación realizada.

\section{Correspondencia:}

Ángela Paredes Aguirre

Universidad Peruana Unión. Carretera Central Km 19.5 Ñaña.

e-mail: angela@upeu.edu.pe,

\section{REFERENCIAS BIBLIOGRÁFICAS}

Aguilar, E. (2008). Evaluación y factores relacionados de las anotaciones de enfermería SEGÚN Modelo SOAPIE, en el Hospital II Vitarte Essalud Lima Perú. (Tesis de Segunda Especialidad).Universidad Nacional Federico Villarreal. Lima.

Alfaro, R. (1995). Aplicación del Proceso de Enfermería. Informe Trad. Ma Teresa Luis Rodrigo.1ra.reimpresion en español. Barcelona España.Mosby-Doyma.p.6-16 i Recuperado de: http://www.seduca2.uaemex.mx/ ckfinder/uploads/files/unidad_1-_lectura_de(2).pdf.

Alfaro, R. (2003). Aplicación del Proceso Enfermero. Fomentar el cuidado en colaboración. 5 ed. Barcelona: Elsevier doyma, S.L; 2003. p. 4. Recuperado de: http://aprendeenlinea.udea.edu.co/lms/moodle/mod/ page/view.php?id=61914

Anglade, C. (2006). Características de las anotaciones de Enfermería y los factores personales e institucionales asociados a su elaboración en el servicio de medicina del Hospital Nacional Dos de Mayo. (Tesis de Bachiller). Universidad Nacional Mayor de San Marcos. Lima. Recuperado://cybertesis.unmsm.edu.pe/handle/ cybertesis/990
Blasceta, R \& Guzmán, A. (2007). Factores que intervienen en la realización del informe de enfermería según la opinión de los enfermeros. (Monografía) .Universidad de Córdova. Recuperado de: ttp://www.enfermeria.fcm. unc.edu.ar/biblioteca/tesis/blascetta_ricardo_jesus.pdf.

Castillo, K. (2011). Relación del nivel de conocimiento $y$ práctica del modelo SOAPIE en la calidad de las anotaciones del profesional de enfermería del Hospital II Vitarte Universidad Peruana Unión. Facultad de Ciencias de la Salud. (Tesis de Bachiller).Lima.

Decreto Supremo Na 004 -2002- SA., 22 de junio del 2002, Diario El Peruano. Ley de Trabajo de la enfermera(o) 27669.

Condezo, M. (2008). La enseñanza aprendizaje y las "anotaciones de enfermería "para una función profesional eficiente. Instituto Nacional Materno Perinatal. Lima (Tesis de Maestría).Universidad Nacional Mayor de San Marcos. Recuperado de: http://cybertesis.unmsm.edu.pe/ handle/cybertesis/1700

Cisneros, F. (2011). Proceso de Atención de Enfermería (PAE). Universidad del Cauca Facultad de ciencias la Salud Programa de Enfermería. Pag 1-23 Recuperado de://artemisa.unicauca.edu.co/pivalencia/archivos/ ProcesoDeAtncionDeEnfermeria- PAE.pdf.

Consejo Internacional de Enfermeras. Definición de Enfermería. Recuperado de: http://www.icn.ch/es/whowe-are/icn-definition-of-nursing/2015

Contreras, M.,Fernández,L., Huapaya,C. \& Medina,N. (2002). Diplomado en Proceso de enfermería. Modulo I. Pag.5-6 .Universidad Peruana Unión. Perú

Chavarry (2013). Introducción a los Modelos y Teorías de Enfermería. Universidad Alas Peruanas. Recuperado de: http://es.slideshare.net/azanero33/modelos-y-teorias-deenfermeria.

Desarrollo del Conocimientos en Enfermería (2014). El modelo de conservación Recuperado de: Recuperaado de http://desarrolloconocimientoenfermeria.blogspot. pe/2013/12/el-modelo-de-la-conservacion.

Desongles, C.J. \& Fernández, R.V. (2004). Diplomado en Enfermería/ATS del Consorcio Hospital General Universitario de Valencia Temario Volumen II. Editorial MAD. Recuperado de: https://books.google.com/ books?isbn $=8466540652$

Doenges, M., Moorhouse, M. (2013). Application of Nursing Process and Nursing Diagnosis

Sexta Edición .FA. Davis Company. Philadelfia. Recuperado de https://es.wikipedia.org/wiki/Enfermería

Dorothy Jhonson (2013). Modelos Enfermeros. Recuperado de: Modelos enfermerossucaldas.blogspot.com/2013/... modelo-del-sistema-conductual.

Ministerio de Salud (2007). Dirección de Calidad Directiva Administrativa para el Proceso de Auditoría de caso de la calidad de la atención en salud. Recuperado de: Modelos www.minsa.gob.pe/.../Auditoria/Directiva Administrativa_Para_Proceso_Auditoria_C. 
Efectividad del programa "Anotaciones, que hablan por ti" en los conocimientos, actitudes y prácticas de los profesionales de enfermería en las anotaciones de enfermería según el modelo SOAPIE en los servicios de hospitalización del Hospital II Vitarte, Lima

Fretel, V.(2004). Evaluación de las Anotaciones de Enfermería durante los años 2000-2002 en el servicio de Neurología del Hospital Guillermo Almenara Irigoyen. Lima. (Tesis de Bachiller). Universidad Nacional Mayor de San Marcos. Recuperado de: http://www.cybertesis. unmsm.edu.pe/sisbib/2004/fretel_mm/pdf/fretel_mmTH.3.pdf

García, J. \& Sánchez. (2013). Diseño e implementación de taller teórico - práctico sobre proceso de enfermería y registro diario de SOAPIE. Revista médico electrónica. Recuperado http:77www.revista -portalesmedicos.com/ revista-medica/proceso-de-enfermeria-registro-diariosoapie

Gutiérrez, J., Esquen, O. \& Gómez,E. (2012). Nivel de Cumplimiento de los registros de enfermería en el servicio de ginecoobstetricia del Hospital Nacional Madre Niño San Bartolomé. Lima. (Monografía). Recuperado de: revistascientificas.upeu.edu.pe/index. $\mathrm{php} / \mathrm{rc} \_$salud/article/download/214/

Hernández- Corral, S. y Hernández -Mayen, L.(2010). Repercusión de un programa de mejora continua en los registros de enfermería en el Instituto de Rehabilitación. México. Enfermería Neurológica, Vol 9, No. 1:13-16, 2010. Recuperado de: http://www.medigraphic.com/ pdfs/enfneu/ene-2010/ene101d.pdf

Hernández, A., \& Guardado, C.(2004). La Enfermería como disciplina profesional Holística. Revista Cubana de Enfermería. Recuperado de: http://bvs.sld.cu/revistas/ enf/vol20_2_04/enf07204.htm

Iyer, P. (1995). Proceso de atención y diagnostico en enfermería. 3era. Edición. Mc Graw-Hill. México.

Kozier, B. (1999). Fundamentos de Enfermería. Conceptos, Proceso Documentos y registros España. Quinta Edición Volumen 1 Editorial Interamericana.

Marcano,M. \& Marcano,N. (2007). Actitud de los estudiantes de los Institutos Universitarios frente a las Tecnologías de la Información y la Comunicación. Universidad Rafael Belloso Chacín - Venezuela. Recuperado de: http://publicaciones.urbe.edu/index.php/telematique/ article/viewArticle/882/2179

Marriner, A. Raile, M. (2007). Modelos y Teorías de Enfermería (sexta edición). Recuperado de:books. google.es/books?id=FLEszO8XGTUC\&printsec=

frontcover\&hl=es

Rogers M. (2015). Historia Enfermería. Recuperado de: www.congresohistoriaenfermeria2015.com/martha.

Montesinos, G. (2002). El conocimiento y la Enfermería. Recuperado de: http:/www.imbiomed.com/1/1/articulos. php? method=showDetail\&id_articulo $=1163 \& i d$ seccion $=161 \&$ id_ejemplar $=152 \& \overline{\text { id }}$ _revista $=33$

Ospina, B., Sandoval, J.,Aristizábal,C. \& Ramírez. (2005). La Escala de Likert en la valoración de los conocimientos y las actitudes de los profesionales de enfermería. Invest Educ Enferm; 23(1): 14-29. Recuperado: http://www. scielo.org.co/pdf/iee/v23n1/v23n1a02.pdf.
Palomino (2009). Calidad de las anotaciones de Enfermería en el Hospital Regional de Ayacucho. (Tesis de Especialidad) Universidad Nacional Mayor de San Marcos. Recuperado de: repebis.upch.edu.pe/cgi-bin/ wxis.exe/

Polit, D. \& Hungler,B. (1987). Investigación Científica en las Ciencias de la Salud. 2da. Edición. Editorial Interamericana.S.a. México.

Potter, P., \& Perry, A. (2002). Fundamentos de Enfermería (Quinta edición ed., Vol1). Madrid. Editorial Harcomt.

Quino, L. (2014). Conocimiento y Actitud sobre notas de Enfermería Modelo SOAPIE en enfermeras del Hospital Regional Manuel Núñez Butrón Puno. (Tesis de Bachiller) Universidad nacional del Altiplano Recuperado de: repositorio.unap.edu.pe/bitstream/handle/UNAP/2571/ Quino_Chata_Lidia.pdf?.

Rayman, B. (2012). Modelos y Teorías en enfermería. Diagnóstico de Enfermería. Recuperado de: .es/2012/05abdellah.htm

Reyes, J., Jara, P., Merino, J. (2007). Adherencia de las enfermeras/os a utilizar un modelo teórico como base de la valoración de enfermería. Chile. Ciencia y Enfermería XIII (1): 45-57, 2007 ISSN 0717-2079 Recuperado de:http://www.scielo.cl/scielo.php?script=sci_ arttext\&pid=S0717-95532007000100006.

Reyes, E. (2015). Abordaje de Enfermería: Proceso de Enfermería. Fundamentos de Enfermería. II Edición. México. Editorial Manual Moderno. Recuperado de: http://es.slideshare.net/ManualModerno/fundamentosde-enfermera-ciencia-metodologa-y-tecnologa-reyesgmez-eva

Rojas, A. (2014). Eficacia de un programa educativo en la calidad técnica de las anotaciones de enfermería en un servicio de medicina interna en un hospital nacional Lima-Perú (Tesis de Bachiller) Universidad mayor de San Marcos. Recuperado de: http://cybertesis.unmsm. edu.pe/xmlui/bitstream/handle/cybertesis/4163/Rojas_ aa.pdf? sequence $=1$

Tataje, M. (2003). Efectividad de un programa educativo en el mejoramiento de conocimiento y calidad de las anotaciones de enfermería en el Hospital Regional de Ica. (Tesis de Especialidad). Universidad Nacional Mayor de San Marcos. Recuperado de: http://cybertesis. unmsm.edu.pe/handle/cybertesis/

Recibido: $15 / 01 / 17$ Aceptado: 10/04/17 